\title{
Schweden und die EU-Ratspräsidentschaft - ehrlicher Makler mit eigenen Interessen
}

\author{
Gunilla Herolf*
}

Schweden übernahm die EU-Ratspräsidentschaft in einer Zeit großer Unsicherheit innerhalb der Union. Ihr Motto „Wir stellen uns der Herausforderung“ machte deutlich, dass man sich der harten Arbeit bewusst war, die die Bewältigung der anstehenden Herausforderungen bedeutete, damit die Union nicht in eine gefährliche Schieflage geriet.

Als die Ratspräsidentschaft am 1. Juli 2009 offiziell begann, war das unsichere Schicksal des Lissabon-Vertrages das zentrale Problem, da das zweite irische Referendum erst zur Halbzeit der Ratspräsidentschaft stattfinden sollte. Alle Vorbereitungen für die Zeit nach dem Referendum mussten unauffällig getroffen werden, um die irischen Wähler nicht zu verärgern. Zu diesem frühen Zeitpunkt waren Probleme im Zusammenhang mit der Umsetzung des Lissabon-Vertrages, die zu einem der wichtigsten Anliegen der Ratspräsidentschaft werden sollten, eher von untergeordneter Bedeutung.

Ein anderes Kernproblem ergab sich aus den unvermeidlichen Verzögerungen bei der Installation eines gut funktionierenden EU-Apparats zu einem Zeitpunkt, als dieser dringend benötigt wurde. Das Europäische Parlament war neu gewählt worden. Die Wiederwahl von José Manuel Barroso dauerte einige Zeit und die Auswahl der neuen Kommissare musste bis nach dem irischen Referendum und der noch ausstehenden Ratifizierung in einigen Ländern warten.

Ein drittes Problem stellte die andauernde Finanzkrise dar. Hieraus folgten für Europa nicht nur Reformen des Finanzsystems, sondern deren wirtschaftliche Auswirkungen erschwerten auch die Art von Reformen, wie sie von Schweden angestrebt worden waren.

Während dieses langen Prozesses, der nahezu die gesamte Ratspräsidentschaft andauerte, galt es, die Europäische Union auf effiziente Weise arbeiten zu lassen, die Ziele zu erreichen, die die Triopräsidentschaft Frankreichs, der Tschechischen Republik und Schwedens sich gesetzt hatten, und insbesondere die spezifisch schwedischen Anliegen auf den Weg zu bringen.

Die Pflichten einer Ratspräsidentschaft umfassen eine große Themenpalette mit einer Vielfalt von Aufgaben. Es ist daher nicht möglich, im Folgenden auf alle Punkte einzugehen. Der Beitrag wird sich auf die Prioritäten der Ratspräsidentschaft konzentrieren, die zu einem großen Teil auch von den Prioritäten der Union bestimmt wurden. Es soll dabei sowohl aus schwedischer wie aus externer Sicht untersucht werden, inwieweit diese Ziele erreicht wurden und welche Bedeutung die erzielten Resultate haben. Darüber hinaus sollen einige grundsätzlichere Überlegungen zur Rolle der Ratspräsidentschaft - etwa als Vermittler und unter dem Aspekt der Führung in der Europäischen Union - sowie zur Frage großer und kleiner Mitgliedstaaten angestellt werden.

\section{Erwartungen an eine Ratspräsidentschaft}

Die Erwartungen anderer Mitgliedstaaten und der restlichen Welt an eine EU-Ratspräsidentschaft haben gewisse gemeinsame Elemente, darunter sind Effizienz, Verlässlichkeit

* Gunilla Herolf, PhD, Senior Researcher, Stockholm International Peace Research Institute (SIPRI), Stockholm. 
und Transparenz im Tagesgeschäft der Ratspräsidentschaft. Andere Faktoren sind schnelles Reagieren und Führungsqualitäten bei unvorhergesehenen Ereignissen. Ein weiteres Kriterium, nach dem Ratspräsidentschaften beurteilt werden, ist, bis zu welchem Punkt sie den Interessen der Europäischen Union Vorrang gegenüber denen ihres eigenen Landes geben.

Bei anderen Feldern kommt unvermeidlich das Element der Subjektivität zum Tragen. So wird das Urteil über die Auswahl der Themen, denen sich die Ratspräsidentschaft verstärkt widmen wollte, oder der Priorität einzelner Themen davon abhängen, wer die Evaluierung vornimmt. Des Weiteren gibt es Grenzen, inwieweit eine Ratspräsidentschaft ihre eigene Agenda bestimmen kann - viele Probleme auf dem Tisch der Ratspräsidentschaften, auch im Falle von Schweden, sind Erblasten früherer Ratspräsidentschaften. Dennoch hat jede Ratspräsidentschaft die Möglichkeit, eigenes Denken und eigene Überzeugungen in Dokumente, vorgeschlagene Kompromisse etc. einzubringen, auch dort, wo es um die tagtägliche EU-Agenda geht. Und schließlich muss bei jeder Bewertung mitberücksichtigt werden, was tatsächlich erreichbar war.

\section{Ziele der schwedischen Ratspräsidentschaft}

Einige Ziele gelten für nahezu alle Ratspräsidentschaften. Wie andere Länder war auch Schweden bemüht, gute Arbeit zu leisten und von anderen als erfolgreich eingeschätzt zu werden. Wie andere Länder hat Schweden sich bemüht, einen spezifischen Eindruck zu hinterlassen und die Europäische Union ein Stück weit in die Richtung zu bewegen, die man sich selbst vorgestellt hat. Ein drittes gemeinsames Anliegen ist, die Bevölkerung des eigenen Landes zu beeinflussen. Das Ziel mag sein, die Europäische Union besser bekannt zu machen und deren Ansehen im eigenen Land zu steigern und/oder zu zeigen, dass die Regierung durch die Ratspräsidentschaft in der Lage war, erfolgreich nationale Interessen zu verfolgen.

Schweden legte die Ziele seiner Ratspräsidentschaft in Zusammenarbeit mit Frankreich und der Tschechischen Republik in einem Achtzehnmonatsprogramm fest, das im Sommer 2008 vorgestellt wurde. Das gemeinsame Programm enthält übereinstimmende Sichtweisen wie auch Kompromissformulierungen, um den unterschiedlichen Ansätzen gerecht zu werden. In mehreren Bereichen lagen die tschechischen und schwedischen Ansichten dichter beieinander als die Ansichten dieser beiden Länder und jene Frankreichs. Die Tschechische Republik und Schweden sind zum Beispiel kritischer gegenüber der Gemeinsamen Agrarpolitik als Frankreich und positiver gegenüber einer EU-Erweiterung über den Westbalkan hinaus. ${ }^{1}$

Ungeachtet aller Vorbereitungen und der Ausarbeitung gemeinsamer Ziele waren die meisten Bereiche, denen die schwedische Ratspräsidentschaft Priorität einräumte, vorgegeben. Sie waren wie das Stockholmer Programm, der Nachfolger des auslaufenden Haager Programms im Bereich Justiz und Inneres, entweder Teil des normalen Geschäftsgangs der Europäischen Union oder wie die Vorbereitungen für den in Kraft tretenden Lissabon-Vertrag übrig geblieben von früheren Ratspräsidentschaften. Andere Fragen im Kontext der wirtschaftlichen Entwicklung und jener des Arbeitsmarkts waren Dauerthemen, auch wenn diese zur Zeit des schwedischen Vorsitzes ebenfalls besondere Aufmerksamkeit erforderten.

Die schwedische Regierung setzte ihre Prioritäten wie folgt: Wirtschaft und Arbeitsmarkt, ein neues Klimaprogramm, das Stockholmer Programm im Bereich Justiz und Inneres, eine EU-Strategie für den Ostseeraum und EU-Erweiterung und Sicherheit. Schlussendlich war die Vorbereitung für das Inkrafttreten des Lissabon-Vertrages, sollte dies während der schwedischen Ratspräsidentschaft geschehen, ein übergeordnetes Ziel. ${ }^{2}$

1 Rat der Europäischen Union: Achtzehnmonatsprogramm des Rates, Dok. 11249/08.

2 Swedish Presidency of the European Union: Prime Minister presents Presidency priorities, 23.06.2009. 


\section{Wirtschaft und Arbeitsmarkt}

Während sich die französische Ratspräsidentschaft mit den sofortigen Antworten auf die Finanzkrise befasst hatte, mussten unter schwedischem Vorsitz wichtige strukturelle Verbesserungen in Angriff genommen werden, um das Vertrauen in die Finanzmärkte wiederherzustellen. Im Herbst verständigten sich die Finanzminister auf einen generellen Ansatz für die Einrichtung neuer Aufsichtsorgane auf der Mikroebene und auf einen „Europäischen Ausschuss für Systemrisiken“, der die Stabilität des Finanzsystems im Ganzen überwachen soll.

Zusätzlich wurden Beschlüsse bezüglich exzessiver Haushaltsdefizite innerhalb des Stabilitäts- und Wachstumspaktes gefasst. Die Minister einigten sich darauf, für neun Mitgliedstaaten ein Verfahren wegen eines übermäßigen Defizits aufgrund eines Haushaltsdefizits von mehr als 3 Prozent des Referenzwertes zu eröffnen. Sie vereinbarten ferner überarbeitete Empfehlungen für vier Mitgliedstaaten, gegen die bereits ein Defizitverfahren lief, und erklärten, dass Griechenland, dessen Defizitverfahren schon im April 2009 eingeleitet worden war, bisher keine effektiven Maßnahmen unternommen habe, um dieses zu einem Abschluss zu bringen. Die Minister einigten sich auch auf die Prinzipien, nach denen der Rückzug aus den Stützungsmaßnahmen für den Finanzmarkt erfolgen soll. ${ }^{3}$

Während der fortdauernden Finanzkrise und der fortschreitenden Globalisierung erforderten Wirtschafts- und Arbeitsmarktfragen im Herbst 2009 besondere Beachtung. Im Zentrum stand die auslaufende Lissabon-Strategie, die 2000 ins Leben gerufen und einmal verlängert worden war. Ihr wird unter dem Namen „Europa 2020“ ein Ansatz nachfolgen, der während der spanischen Ratspräsidentschaft auf den Weg gebracht werden soll. In Abstimmung mit diesem Zeitplan drehten sich die schwedischen Bemühungen um Vorarbeiten in Form von Diskussionen über die neue Strategie, aber auch durch die Vorlage eines Berichtes über nachhaltige Entwicklung, den der Europäische Rat anlässlich seines Treffens am 10./ 11. Dezember 2009 guthieß. Im Bericht fanden sich traditionelle schwedische Politikansätze bezüglich (1) Vollbeschäftigung und integrative Arbeitsmärkte, (2) Gesundheitsförderung für die alternden Arbeitskräfte in der Europäischen Union, vor allem durch Aushandlung der Richtlinien für Patientenmobilität und elektronische Gesundheitsdienste sowie durch Fortschritte im pharmazeutischen Bereich und (3) Betonung der Bedeutung der Gleichberechtigung der Geschlechter für Wirtschaftswachstum und Beschäftigung. ${ }^{4}$ Der Bericht zur Nachhaltigkeitsstrategie stimmte mit dem Bericht der Kommission zu diesem Thema überein. ${ }^{5}$

Trotz der Tatsache, dass alle 27 Mitgliedstaaten der Europäischen Union den Bericht zur Nachhaltigkeitsstrategie akzeptierten, wird der nächste Schritt in diesem Prozess schwierig werden. Es muss daran erinnert werden, dass es sich um Langzeitziele handelt, sie also nicht Entscheidungen über eine konkrete Umsetzung beinhalten, und dass die Gegner dieser Ziele ihre Sichtweise erst dann öffentlich machen werden, wenn diese Stufe erreicht wird.

3 Swedish Presidency of the European Union: ECOFIN Council agrees on new financial supervisory architecture, 02.12.2009. Die neun Staaten waren Belgien, Deutschland, Italien, die Niederlande, Österreich, Portugal, die Slowakei, Slowenien und die Tschechische Republik. Die vier Staaten waren Großbritannien, Frankreich, Irland und Spanien.

4 Rat der Europäischen Union: Überprüfung der EU-Strategie für nachhaltige Entwicklung (2009) - Bericht des Vorsitzes, Dok. 16818/09: EurActiv: Schweden drängen zu ,skandinavischem Touch` bei Lissabon-Strategie II, 13.07.2009.

5 Europäische Kommission: Mitteilung der Kommission an das Europäische Parlament, den Rat, den europäischen Wirtschafts- und Sozialausschuss und den Ausschuss der Regionen: Förderung einer nachhaltigen Entwicklung durch die EU-Politik: Überprüfung der EU-Strategie für nachhaltige Entwicklung 2009, KOM (2009) 400. 
Das plötzliche Zurückziehen ihres Haushaltsentwurfs, kurz bevor die Europäische Kommission ihn im Europäischen Parlament vorstellen wollte, war in diesem Zusammenhang eine große Enttäuschung für Schweden. Die damalige Ministerin für europäische Angelegenheiten, Cecilia Malmström, nannte diese Verzögerung in ihrem Blog „bemerkenswert und bedauerlich, da wir wirklich diskutieren müssen, wie wir die vor uns liegenden Herausforderungen angehen - und finanzieren - wollen. Jetzt, da wir in wenigen Wochen einen neuen Vertrag haben werden, brauchen wir auch einen neuen modernen Haushalt. "6 Für Schweden war ein neuer Haushalt einschließlich einer substanziellen Reduzierung der Gemeinsamen Agrarpolitik seit Jahren eine drängende Notwendigkeit, um mehr Spielraum für Investitionen und Bildung zu gewinnen.

\section{Ein neues Klimaprogramm}

Eine weitere Priorität war für Schweden ein neues Klimaprogramm, das vom Klimagipfel in Kopenhagen im Dezember 2009 beschlossen werden sollte, das alle Länder einschlieBen und umfassende Vorhaben zur Reduzierung von Emissionen enthalten sollte, sodass das $2{ }^{\circ} \mathrm{C}$-Ziel erreicht werden konnte. ${ }^{7}$

Über den Herbst war es die vornehmste Aufgabe für Schweden, eine einheitliche Position der Europäischen Union zu erreichen, was aber wegen der wirtschaftlichen Schwierigkeiten in einigen Ländern nicht einfach war. In diesem Zusammenhang wurde Deutschland als wichtiger Bündnispartner betrachtet, da es die gleichen ehrgeizigen Ziele für Reduzierungen auf nationaler Ebene hatte wie Schweden.

Die noch schwierigere Aufgabe für die Ratspräsidentschaft stellte der Versuch dar, die USA und einige andere Länder, vor allem China - diese beiden Staaten produzieren gemeinsam die Hälfte aller Treibhausgase - dazu zu bewegen, ihr Engagement in dieser Frage zu verstärken. Die USA wurden um eine gesetzlich verbindliche und die gesamte Wirtschaft betreffende Verpflichtung bei der Reduzierung der Emissionen ersucht, während China um verbindliche Maßnahmen gebeten wurde. China legte zwar ein vages Angebot vor, das aber eine relative Größe vorsah und sich damit am Wirtschaftswachstum orientierte, und das USamerikanische Reduzierungsangebot erwies sich als bescheiden, denn Präsident Barack Obamas Zusicherung einer Reduzierung um 17 Prozent für die Jahre 2005 bis 2020 bedeutete lediglich eine Reduzierung von 4 Prozent seit 1990. Da die Europäer angeboten hatten, ihre eigenen Reduzierungen von 20 auf 30 Prozent für die Zeit nach 2012 zu steigern, ,,sofern sich andere Industrieländer zu vergleichbaren Emissionsreduzierungen und die Entwicklungsländer zu einem ihren Verantwortlichkeiten und jeweiligen Fähigkeiten angemessenen Beitrag verpflichten“, 8 verblieb das europäische Angebot auf dem niedrigeren Niveau.

Positiv zu vermerken ist, dass die Zusage der Europäischen Union von insgesamt 7,2 Milliarden Euro als Hilfe für die am wenigsten entwickelten und gefährdetsten Länder für Anpassungsmaßnahmen an den Klimawandel zwischen 2010 und 2012 großzügiger ausfiel als zuvor angenommen. Schweden hatte frühzeitig 800 Millionen Euro zugesagt, um andere zum Nachziehen zu ermuntern. Während dies der höchste Pro-Kopf-Beitrag war, waren drei andere Beiträge in absoluten Zahlen höher: Großbritannien mit 1,65 Milliarden Euro sowie Frankreich und Deutschland mit jeweils 1,26 Milliarden Euro. ${ }^{9}$

6 Cecilia Malmström, Ministerin für europäische Angelegenheiten: Strategy talk in Brussels, in: Swedish Presidency of the European Union. EU Minister's Blog, 17.11.2009, abrufbar unter: http://se2009.wordpress.com/ 2009/11/17/strategy-talk-in-Brussels/ (letzter Zugriff: 20.03.2010).

7 Sie dazu Severin Fischer/Jo Leinen: Zwischen Führungsrolle und Sprachlosigkeit: Europas Lehren aus dem Klimagipfel in Kopenhagen, in diesem Heft, S. 117-130.

8 Rat der Europäischen Union Tagung des Europäischen Rates in Brüssel vom 18./19. Juli 2009. Schlussfolgerungen des Vorsitzes, Dok. 11225/09, S. 11.

9 Anne Eckstein: EU pledges 7.2 bn euro to aid developing countries, in: Europolitics, 11.12.2009. 
Das endgültige Ergebnis der Konferenz, die „Übereinkunft von Kopenhagen“, die von mehr als 30 Ländern ausgehandelt und von den anwesenden Ländern ,zur Kenntnis genommen“ wurde, stellte eine große Enttäuschung für die schwedische Ratspräsidentschaft dar. Ohne rechtsverbindliche Zusagen war die Vereinbarung für Umweltminister Andreas Carlgren zutiefst unbefriedigend. Der Minister, der dies als ein zentrales Anliegen der gesamten Ratspräsidentschaft betrachtet hatte, nannte die Vereinbarung bei dem darauffolgenden Treffen der Umweltminister „ein Desaster und ein wirklich großes Scheitern, aus dem wir werden lernen müssen“. ${ }^{10}$

Die Reaktionen in Schweden waren ziemlich gleichlautend. Die meisten Kommentatoren schoben die Schuld auf die USA und China, die nicht gewillt gewesen seien, substanzielle Reduzierungen zuzusagen, und auf einige andere Länder, die die Konferenz mit endlosen Verfahrensfragen blockiert hätten. Das Scheitern wurde nicht auf einen Konflikt zwischen Arm und Reich zurückgeführt, da viele der armen Länder auf der Seite der Europäischen Union standen. Einige Nichtregierungsorganisationen kritisierten auch die schwedische Ratspräsidentschaft, indem sie argumentierten, dass während der Konferenz mehr hätte getan werden können, um zu einem Abkommen zu kommen.

\section{Das Stockholmer Programm}

Schweden musste das Stockholmer Programm als Aufgabe übernehmen, weil das Haager Programm von 2004 auslief. ${ }^{11}$ Aber das Programm umfasst auch Themen, mit denen sich Schweden innerhalb der Europäischen Union profilieren möchte. Das Europäische Parlament hatte das Programm nach einigen geringfügigen Änderungen mit großer Mehrheit angenommen, bevor es vom Europäischen Rat auf seinem Gipfeltreffen am 10./11. Dezember 2009 beschlossen wurde. $^{12}$

Das Stockholmer Programm umfasst viele Bereiche, wie zum Beispiel die Zusammenarbeit von Polizei und Zollbehörden, Katastrophenschutz, Zusammenarbeit im Straf- und Zivilrecht, Asylpolitik, Migration und Visapolitik. Dabei handelt es sich vielfach um den Ausgleich verschiedener Politikansätze. Einerseits versucht das Programm, die Verbrechensbekämpfung durch grenzüberschreitende Zusammenarbeit und Informationsaustausch effektiver zu machen. Auf der anderen Seite stehen Maßnahmen, die die Rechte von Verbrechensopfern und Verdächtigen garantieren sollen. Diese Punkte betreffen die Bereiche der persönlichen Unversehrtheit und Sicherheit, wobei diskutiert wurde, ob die persönliche Integrität unter den Bemühungen, Sicherheit zu schaffen, gelitten hat.

Zentrale Fragen für ganz Europa, die auch heiß umstritten sind, betreffen Einwanderung, Asylpolitik und Grenzschutz. Vor allem die Mittelmeerländer haben sich in dieser Debatte engagiert angesichts der großen Zahl von Flüchtlingen, die in diesen Ländern ankommen.

10 United Nations Framework Convention on Climate Change: Copenhagen Accord, Decision -/CP.15, advance unedited version, 19.12.2009, abrufbar unter: http://unfccc.int/files/meetings/cop_15/application/pdf/cop15_ cph_auv.pdf (letzter Zugriff: 20.03.2010); European Voice: Commission to review Copenhagen failings, 22.12.2009.

11 Vgl. Jörg Semmler: Das Instrument der Mehrjahresprogramme in der europäischen Justiz- und Innenpolitik, in: integration 1/2009, S. 63-74.

12 Die Abgeordneten des Europäischen Parlaments konnten keine Einigung über die Rechte von Homosexuellen erreichen und italienische Abgeordnete des Europäischen Parlaments erstritten zusätzliche Gelder für überfüllte Gefängnisse. EurActiv: Parliament approves Stockholm Programme, with Prison Add-Ons, 26.11.2009; Swedish Presidency of the European Union: Debate in Strasbourg on the Stockholm Programme, 25.11.2009; Rat der Europäischen Union: Entwurf eines Mehrjahresprogramms für einen Raum der Freiheit, der Sicherheit und des Rechts im Dienste der Bürger (Stockholmer Programm), Dok. 14449/09. 
Die schwedische Auffassung ist dadurch geprägt, dass das Land eine verhältnismäßig große Zahl an Flüchtlingen aufnimmt. Schweden hat jahrelang argumentiert, dass die Lasten gleichmäßiger auf alle EU-Mitgliedstaaten verteilt werden müssen, indem ein gemeinsames Flüchtlings- und Asylsystem geschaffen wird. Diese Ansicht vertritt auch die Kommission.

Im Stockholmer Programm werden die drei zentralen Bereiche wie folgt angesprochen, um diese in ein Gleichgewicht zu bringen:

- Förderung von Mobilität und legaler Einwanderung,

- Hilfe für Schutzbedürftige,

- Grenzmanagement an den Außengrenzen.

Die Förderung von Mobilität und legaler Einwanderung wird von Schweden als eine zwingende Notwendigkeit für ein Europa betrachtet, dessen Bevölkerung abnimmt und das daher Zuwanderung von Arbeitskräften benötigt, um seine Gesellschaft zu erhalten. Gleichzeitig sollte diese Politik eng vernetzt werden mit Bemühungen, die Entwicklung in den Herkunftsländern zu fördern. ,Brain drain` ist eine reale Gefahr für Entwicklungsländer. Dies soll durch ,zirkuläre Migration ‘ vermieden werden, das heißt die Zeit, die Migranten in der Europäischen Union verbringen, soll schlussendlich den Herkunftsländern zugute kommen. Diese Politik muss auch mit effektiven Maßnahmen gegen illegale Einwanderung gekoppelt werden, mit Vorrang für den Kampf gegen Menschenhandel.

Ein weiterer Punkt ist Hilfe für Menschen in Not, die in steigenden Zahlen in behelfsmäBigen Booten, Containern oder mit anderen menschenunwürdigen Hilfsmitteln in Europa eintreffen. Eine Weigerung, diese Flüchtlinge aufzunehmen, würde unvermeidlich zu vermehrtem Menschenschmuggel führen. Das Stockholmer Programm baut auf dem „Europäischen Pakt zu Einwanderung und Asyl“ auf, den der Europäische Rat im Oktober 2008 beschlossen hat, der auch die Idee eines Europäischen Unterstützungsbüros für Asylfragen einschließt. ${ }^{13}$ Ziel des Europäischen Unterstützungsbüros für Asylfragen ist die Koordination Kapazitäten bildender Bemühungen, um die Einrichtung eines Gemeinsamen Europäischen Asylsystems bis $2012 \mathrm{zu}$ fördern. Auch ein gemeinsames Rückführungsprogramm wird im Stockholmer Programm befürwortet. Die Kooperation mit Drittländern, in denen große Flüchtlingsgruppen leben, soll Flüchtlingen helfen, die keine Möglichkeit haben, mit eigenen Mitteln in die Europäische Union zu gelangen.

Der Schutz der Außengrenzen schließt eine Verstärkung der Grenzkontrollen ebenso ein wie die Möglichkeit für diejenigen, nach Europa einzureisen, die dieses Recht haben. Dies bedeutet verstärkte Kooperation und Informationsaustausch durch die Mitgliedstaaten und Frontex ${ }^{14}$ sowie die Nutzung neuer Technologien.

Nach Ansicht der schwedischen Ratspräsidentschaft hängen diese drei Themen zusammen. Das Ziel ist, dass Europa mit einer fairen und transparenten Politik für legale Einwanderung und der Hilfe für Menschen in Not eine geringere Last bei Kontrollen der illegalen Einwanderung hätte.

Das Stockholmer Programm ist trotz der Akzeptanz durch alle 27 Mitgliedstaaten noch nicht gesichert, da es nur ein Programm ist und keine verbindlichen Beschlüsse enthält. Nicht alle teilen die Ideen hinter dem Stockholmer Programm und die, die dagegen sind,

13 Rat der Europäischen Union: Europäischer Pakt zu Einwanderung und Asyl, Dok. 13189/08.

14 Frontex (abgeleitet von ,frontières extérieures`) ist die ,Europäische Agentur für die operative Zusammenarbeit an den Außengrenzen der Mitgliedstaaten der Europäische Union“, sie ist eine spezialisierte und unabhängige Einrichtung, mit der Aufgabe, die operationelle Zusammenarbeit zwischen den Mitgliedstaaten im Bereich der Grenzsicherung zu koordinieren. Vgl. Verordnung (EG) Nr. 2007/2004 des Rates vom 26. Oktober 2004 zur Errichtung einer Europäischen Agentur für die operative Zusammenarbeit an den Außengrenzen der Mitgliedstaaten der Europäischen Union, in: Amtsblatt der EU, Nr. L 349 vom 25. November 2004, S. 1-11. 
werden sicherlich versuchen, die Implementierung zu verhindern. Den südeuropäischen Ländern gehen die Bestimmungen des Stockholmer Programms gegen illegale Einwanderung nicht weit genug. Viele halten die Chancen, dass eine gemeinsame Asylpolitik beschlossen wird, für gering, da viele Länder nicht bereit sind, mehr Flüchtlinge aufzunehmen. Auch sehen sie nicht die Notwendigkeit einer Arbeitsmigration nach Europa. Es ist außerdem ein Bereich, in dem parteipolitische Orientierungen von Bedeutung sind: Das Programm wird vor allem von den Liberalen gestützt, von Nichtregierungsorganisationen aber als nicht weit genug gehend eingeschätzt. ${ }^{15}$ Aus diesen Gründen wird es für die spanische Ratspräsidentschaft schwierig sein, diese Themen weiterzuverfolgen. Die Kommission hat sich bereits auf die Linie des Stockholmer Programms festgelegt und schwedische Positionen werden weiter Eingang in die Kommissionsberatungen finden, da Cecilia Malmström zur neuen Kommissarin für Inneres ernannt wurde.

\section{Die EU-Strategie für den Ostseeraum}

Die EU-Strategie für den Ostseeraum ist, anders als die zuvor behandelten Initiativen, weder eine Erblast früherer Ratspräsidentschaften noch ein besorgniserregendes Thema für ganz Europa. Diese Strategie betrifft nur Schwedens direkte Nachbarschaft, doch es ist geplant, dieser Initiative eine ähnliche für den Donauraum folgen zu lassen. Auch die Alpenregion und der Mittelmeerraum wurden in diesem Kontext erwähnt.

Die EU-Strategie für den Ostseeraum ist das erste Beispiel innerhalb der Europäischen Union für eine sogenannte ,makroregionale Kooperation', wo man sich mit einer umfassenden Strategie, die mehrere EU-Politikbereiche bündelt, auf eine einzige Region konzentriert. Auch wenn sie, zumindest zu diesem Zeitpunkt, noch keine zusätzlichen Gelder vorsieht, bedeutet sie doch eine Zusammenführung europäischer und regionaler Ressourcen, die nicht nur Regierungen, sondern auch weitere Akteure einschließt, um maximale Wirkung zu erzielen. Zusätzlich stellt sie auch ein Auffangbecken verschiedener Herausforderungen und Gelegenheiten dar, Ambitionen zu bündeln. Die Strategie sieht eine enge Zusammenarbeit mit Russland wie auch mit Norwegen und Weißrussland vor.

Die EU-Strategie für den Ostseeraum wurde aus der Nördlichen Dimension der europäischen Zusammenarbeit weiterentwickelt. Im Dezember 2007 bat der Europäische Rat die Kommission, für diese Region eine Strategie mit Schwerpunkt auf den umweltpolitischen Herausforderungen für den Ostseeraum zu entwickeln. Die Strategie wurde am 10. Juni 2009 vorgestellt und schließlich am 29./30. Oktober 2009 vom Europäischen Rat beschlossen. Sie soll im Oktober 2010 mit einem Treffen der Beteiligten in Tallinn in Kraft gesetzt werden. Die Kommission soll im Juni 2011 einen Fortschrittsbericht vorlegen. ${ }^{16}$

Die Idee der EU-Strategie für den Ostseeraum speist sich aus einer Kombination umweltpolitischer Probleme der Ostsee und der ungleichen wirtschaftlichen Entwicklung innerhalb dieser Region, in der acht von neun Anrainerstaaten Mitglied der Europäischen Union sind. Diese Probleme können nur gemeinsam durch Zusammenarbeit und weitere Integration innerhalb der Region angegangen werden. Dabei sind die folgenden vier zentralen Herausforderungen zu bewältigen:

15 EurActiv: Parliament split on 'progressive' Swedish immigration programme, 12.10.2009.

16 Cecilia Malmström, Ministerin für europäische Angelegenheiten: An EU Strategy for the Baltic Sea region, Rede vor dem Europäischen Parlament in Straßburg, 12.12.2007, abrufbar unter: www.regeringen.se/sb/d/ 3211/a/94598 (letzter Zugriff: 20.03.2010); Rat der Europäischen Union: Tagung des Europäischen Rates vom 14. Dezember in Brüssel. Schlussfolgerungen des Vorsitzes, Dok. 16616/07; Rat der Europäischen Union: Tagung des Europäischen Rates vom 29./30. Oktober 2009. Schlussfolgerungen des Vorsitzes, Dok. 15265/09, Punkt 35-36. 
1. Nachhaltigkeit im Umweltbereich: Die Umwelt steht im Zentrum der Strategie. Die Ostsee ist verschmutzt und wegen ihrer geringen Tiefe und der sehr schmalen Verbindung mit der Nordsee besonders gefährdet. Die Prioritäten des Aktionsplans konzentrieren sich darauf, den Gebrauch und die Auswirkungen von gefährlichen Stoffen zu reduzieren und zu einer Modellregion für saubere Schifffahrt zu werden.

2. Wohlstand in der Region: Bemühungen, die wirtschaftliche Entwicklung der Region zu fördern, umfassen vor allem den Abbau von Handelshemmnissen und die Weiterführung von Reformen. Der Abbau von Handelshemmnissen schließt die volle Anwendung der EU-Gesetzgebung ein, vor allem die Anwendung der Regeln des Europäischen Binnenmarktes, die als besonders wichtig für kleine und mittlere Unternehmen angesehen werden. Innovation soll durch Wissens- und Kompetenztransfer innerhalb der Region gefördert werden, mit dem Ziel, Polen und die Baltischen Staaten dabei zu unterstützen, zu den anderen Staaten aufzuschließen.

3. Zugang und Attraktivität der Region: Hier geht es vor allem um die Punkte Transport und Energie. Die Größe der Region mit schwierigen geografischen und klimatischen Gegebenheiten und mangelnder Infrastruktur erfordert Verbesserungen im Transportwesen. Der Aktionsplan befasst sich außerdem mit den benötigten Verbesserungen im Energiesektor, dem es an adäquater Infrastruktur fehlt und der innerhalb der Region besser vernetzt werden muss.

4. Sicherheit innerhalb der Region: In diesem Zusammenhang sind zwei besondere Herausforderungen relevant, einerseits die Seeschifffahrt und dabei vor allem der Transport von Öl und Flüssiggas und andererseits die Notwendigkeit der grenzüberschreitenden Verbrechensbekämpfung.

Insgesamt sind 15 Schwerpunktthemen im Aktionsplan aufgelistet, von denen jedes einem Mitgliedstaat zugewiesen ist. Die Schwerpunkte sind ihrerseits in 76 konkrete Projekte aufgeteilt, die detaillierte Angaben zu Ländern, führenden Partnern, Zielen und Prüfterminen enthalten. ${ }^{17}$

Die EU-Strategie für den Ostseeraum wurde von allen Ländern unterstützt, nicht nur von den Anrainerstaaten, und wird im Allgemeinen für eine gute Idee für die weitere Entwicklung der Region gehalten, die den Herausforderungen gerecht wird. Der problemlose Durchmarsch durch die Instanzen wurde vermutlich durch die frühe deutsche Rückendeckung für die Strategie erleichtert. Wichtig für den schlussendlichen Erfolg ist, wie auch Kommentatoren innerhalb der Kommission betont haben, dass die Strategie auch weiterhin auf höchster Ebene unterstützt wird, damit sie ein kraftvolles Mittel für die gesetzten Ziele bleibt. Ein weiterer zentraler Punkt wird sein, wie Russland auf substanzielle Weise in die Aktivitäten eingebunden werden kann. ${ }^{18}$

17 Europäische Kommission: Mitteilung der Kommission an das Europäische Parlament, den Rat, den Europäischen Wirtschafts- und Sozialausschuss und den Ausschuss der Regionen der Europäischen Gemeinschaften zur Strategie der Europäischen Union für den Ostseeraum, KOM (2009) 248; Europäische Kommission: Commission Staff Working Document, Accompanying the Communication from the Commission to the European Parliament, the Council, the European Economic and Social Committee and the Committee of the Regions concerning the European Union Strategy for the Baltic Sea Region. Action Plan, SEC (2009) 712.

18 Angela Merkel/Fredrik Reinfeldt: Ökat Östersjösamarbete i EU [Herausforderungen rund um die Ostsee], in: Svenska Dagbladet, 04.02.2009. Rikard Bengtsson: An EU Strategy for the Baltic Sea Region: Good Intentions Meet Complex Challenges, Swedish Institute for European Policy Studies (SIEPS): European Policy Analysis 9/2009. 


\section{EU-Erweiterung und Sicherheitsfragen}

Sicherheitsfragen stellen für eine Ratspräsidentschaft am ehesten den Prüfstein für ihre Fähigkeit dar, auf unerwartete Situationen zu reagieren. In dieser Hinsicht hatte Schweden Glück. Während der Krieg in Georgien in die Zeit der französischen Ratspräsidentschaft fiel und die Tschechische Republik sowohl im russisch-ukrainischen Gasstreit und im GazaKonflikt vermitteln musste, konnte sich Schweden stattdessen an den Altlasten versuchen, die die Europäische Union seit Jahren vor sich her schob. Dies war die Aufgabe von Außenminister Carl Bildt.

\section{Erweiterung}

Einige dieser Altlasten betrafen die EU-Erweiterung, ein Bereich, in dem Schweden an seine Ratspräsidentschaft den Anspruch stellte, den Prozess trotz der unterschiedlichen Probleme bei den betroffenen Ländern und der Erweiterungsmüdigkeit unter den aktuellen Mitgliedstaaten voranzutreiben. Eines der dringlichsten dieser Probleme war der Grenzkonflikt zwischen Slowenien und Kroatien, der die EU-Beitrittsverhandlungen mit Kroatien blockierte. Die schwedische Ratspräsidentschaft half bei der Vereinbarung zwischen den beiden Ländern mit, ein Schiedsverfahren zu beginnen, und am 4. November 2009 trafen sich die Premierminister von Kroatien und Slowenien in Stockholm, um diese Vereinbarung zu unterzeichnen. In das Kapitel Erweiterung fiel auch das Thema der Visafreiheit, das formal in den Bereich Justiz und Inneres gehört. Hier gab es im Juli 2009 einen Fortschritt, als den Bürgern von Mazedonien, Montenegro und Serbien gestattet wurde, sich ab dem 19. Dezember 2009 im größten Teil Europas frei bewegen zu können. ${ }^{19}$

Zwei andere, die EU-Erweiterung betreffende Themen konnten nicht so gut gelöst werden. Bei dem ersten ging es um die Bemühungen, die Differenzen zwischen Griechenland und (der ehemaligen jugoslawischen Republik) Mazedonien über einen akzeptablen Namen für Letztere beizulegen. Das zweite betraf den Konflikt zwischen Zypern und der Türkei, da zypriotischen Schiffen und Flugzeugen immer noch die Nutzung türkischer Häfen und Flughäfen untersagt ist.

All diese Themen waren für Schweden besonders wichtig wegen Schwedens und nicht zuletzt Außenminister Carl Bildts starkem Interesse an einer fortgesetzten Erweiterung der Europäischen Union. Dieser Prozess kam während der schwedischen Ratspräsidentschaft etwas weiter voran. Für den kroatischen Beitritt war das Abkommen mit Slowenien wichtig und so konnten neun von 35 Kapiteln der Beitrittsverhandlungen im Herbst 2009 abgeschlossen werden. Die Verhandlungen befinden sich inzwischen in der Schlussphase. Im Juli 2009 stellte Island seinen Aufnahmeantrag, Serbien folgte im Dezember 2009. Dazu kommen noch die früher gestellten Beitrittsanträge von Albanien (April 2009) und Montenegro (Dezember 2008). Island wird die Verhandlungen vermutlich bereits im Frühjahr 2010 aufnehmen. ${ }^{20}$

Im Oktober 2009 schlug die Kommission die Aufnahme von Verhandlungen mit Mazedonien, das bereits Kandidatenstatus hatte, vor. Für den damaligen Erweiterungskommissar Olli Rehn war diese Entscheidung ,eine sehr starke Ermutigung, die Namensfrage zu lösen und damit von der Agenda zu löschen“. Trotz dieser Mahnung war keine Einigung möglich. Da dies aber die Voraussetzung für die griechische Zustimmung zur Eröffnung von Beitritts-

19 Delegation of the European Union to the United Nations: Croatia and Slovenia agreed on border issue - EU closer to enlargement, 04.11.2009; EurActiv: Balkan-EU Visum als „großer Schritt für Mazedonien“ angesehen, 16.07.2009.

20 Swedish Presidency of the European Union: Serbia applies for EU membership, 22.12.2009. 
verhandlungen war, musste der Europäische Rat die Entscheidung über die Festlegung eines Datums für den Beginn von Beitrittsverhandlungen verschieben. ${ }^{21}$

Der Beitritt der Türkei ist trotz der Tatsache, dass das Land Kandidatenstatus hat, unter den Mitgliedstaaten umstritten. Der schwedische Standpunkt heißt keineswegs, dass die türkische Mitgliedschaft bedingungslos oder schnell zu erreichen wäre. Cecilia Malmström sagte: „Wir unterstützen die Mitgliedschaft - wenn die Türkei die Kriterien erfüllt -, weil wir glauben, dass eine demokratische und offene Türkei viel zur EU beitragen kann und dass das Land als wichtige Brücke zwischen Europa und der islamischen Welt dienen kann. Eine Mitgliedschaft liegt natürlich noch weit in der Zukunft, aber es ist wichtig, dass wir positive Signale an die Türkei aussenden und an alle, die den Premierminister und die Regierung dazu bewegen wollen, die EU anzusprechen und das Land in die EU zu führen. “22

Während der Ratspräsidentschaft richtete sich die schwedische Politik nicht darauf, andere Mitgliedstaaten von den Vorteilen einer türkischen Mitgliedschaft zu überzeugen. Dies wurde bereits während der ersten Tage der Ratspräsidentschaft im Zusammenhang mit dem Besuch des französischen Präsidenten Nicolas Sarkozy in Stockholm deutlich. Türkische Reformen wurden unterstützt, während die Türkei durchgängig gedrängt wurde, den 2005 mehr oder weniger gestoppten Reformprozess wieder aufzunehmen. Im Herbst 2009 erlebte man sowohl Fortschritte - Friedensabkommen mit Armenien im Oktober - wie auch Rückschläge - das gerichtliche Verbot der kurdischen „Partei der demokratischen Gesellschaft“ (DTP) im Dezember. Am 21. Dezember 2009 wurden die Verhandlungen zum Kapitel Umwelt aufgenommen, laut Zeitungsberichten nach starkem Druck von Carl Bildt. ${ }^{23}$

\section{Die Östliche Partnerschaft}

Obwohl die Östliche Partnerschaft formell nicht Teil der schwedischen Ratspräsidentschaft war, so war sie doch zeitlich gut mit ihr verknüpft und auch mit den schwedischen Ambitionen bei der Erweiterung vernetzt, da die Östliche Partnerschaft den teilnehmenden Staaten die Chance bietet, sich für eine Mitgliedschaft zu empfehlen.

Die Östliche Partnerschaft wurde - als Fortführung der traditionellen schwedischen Politik - zum ersten Mal als polnisch-schwedischer Vorschlag vorgestellt und im Mai 2009 als eine EU-Politik eingeführt. ${ }^{24}$ In ihrem Mittelpunkt stehen Armenien, Aserbaidschan, Georgien, Moldova, die Ukraine und Weißrussland (wenn die Lage in Weißrussland dies ermöglicht). Projekte des Europäischen Nachbarschafts- und Partnerschaftsinstruments können auch auf Russland ausgedehnt werden. Einer der Gründe für diese Initiative war, der Europäischen Nachbarschaftspolitik mehr Nachdruck zu verleihen. Ein weiterer Grund lässt sich am Zeitpunkt ablesen, zu dem der ursprüngliche Vorschlag gemacht wurde, nämlich im Jahr 2008 als der französische Vorschlag für eine Union für das Mittelmeer lanciert wurde. ${ }^{25}$

21 EUbusiness: EU urged to start Macedonia entry talks: Commission, 14.10.2009; World News Connection (WNC): Macedonian Information Agency (MIA): Macedonia. Protesters Demonstrate 'Enormous Disappointment' with EU Decision, 12.01.2010.

22 Cecilia Malmström, Ministerin für europäische Angelegenheiten: Turkiet och EU [Die Türkei und die EU], Europaforum, Hässleholm 8. April 2008, abrufbar unter: www.regeringen.se/sb/dd//10173/a/102489 (letzter Zugriff: 20.03.2010).

23 Ingrid Hedström: Barroso svår nöt för Reinfeldt [Barroso schwierige Frage für Reinfeldt], in: Dagens Nyheter, 03.07.2009; EurActiv: EU criticizes Turkey's court ban of Kurdish party, 15.12.2009.

24 Vgl. Katrin Böttger: Im Osten nichts Neues? Ziele, Inhalte und erste Ergebnisse der Östlichen Partnerschaft, in: integration 4/2009, S. 372-387.

25 Es gab einige grundlegende Unterschiede zwischen dem polnisch-schwedischen Vorschlag vom 26. Mai 2008 und dem Vorschlag für eine Union für das Mittelmeer. Einer davon war, dass die Östliche Partnerschaft innerhalb der Europäischen Union geschaffen werden sollte, nicht parallel zu ihr. Siehe: Polish-Swedish Proposal. Eastern Partnership, 23.05.2008, abrufbar unter: www.tepsa.eu/docs/draft_proposal_eastern_partnership.pdf (letzter Zugriff: 20.03.2010). 
Im Zentrum des Interesses standen zwei spezielle EU-Politikfelder, die die Länder der Östlichen Partnerschaft am schnellsten realisiert sehen wollten: visumfreies Reisen und Freihandelszonen. Beide wurden jedoch , verwässert', bevor sie von der Europäischen Union beschlossen wurden. Freihandelszonen waren nicht akzeptabel für die EU-Länder, vor allem für Frankreich, die diese als direkte Konkurrenz zu ihren eigenen landwirtschaftlichen Produkten sahen. Die Einführung von Freihandelszonen wurde auf eine spätere Stufe der Östlichen Partnerschaft verschoben. Visumfreies Reisen wurde von einigen Staaten, darunter Deutschland, als ein potenzieller Weg auch für illegale Arbeitskräfte und für Kriminelle in die Europäische Union betrachtet, sodass der Wortlaut des Abschlussdokumentes sehr vorsichtig formuliert wurde. ${ }^{26}$ Die diesbezüglichen schwedischen Bemühungen während der Ratspräsidentschaft waren somit darauf beschränkt, den Visumsprozess zu erleichtern und zu entbürokratisieren.

Ereignisse innerhalb und außerhalb der Europäischen Union wurden für die Östliche Partnerschaft ebenfalls zu einem Problem. Der Georgien-Konflikt 2008 veränderte die Wahrnehmung der Östlichen Partnerschaft, die viele europäische Staaten fast schon als ein Bollwerk gegen Russland - also weit von ihrer ursprünglichen Idee entfernt - verstanden. Auf russischer Seite hatte eine gesteigerte Empfindlichkeit gegenüber dem westlichen Einfluss auf die ehemaligen Sowjetrepubliken dazu geführt, die Europäische Union ähnlich zu betrachten, wie lange Zeit die NATO gegolten hatte. Ein weiterer negativer Einfluss auf die Östliche Partnerschaft resultierte aus der unruhigen politischen Entwicklung in einigen der sechs Länder in der jüngsten Zeit. Auf dem ersten Außenministertreffen der Östlichen Partnerschaft am 8. Dezember 2009 fassten die Minister das bisher Erreichte zusammen. Sie begrüßten die Fortschritte bei den sogenannten ,Flagschiff-Initiativen', identifizierten aber auch die Notwendigkeit für weitere Fortschritte bei den Verhandlungen über Freihandelszonen wie auch für die Mobilität der Bürger und für die Bereitstellung weiterer Gelder. ${ }^{27}$

\section{Schweden und die Bündnisfreiheit}

Bevor die schwedischen Vorschläge zu Sicherheit und Verteidigung beschrieben werden, soll noch auf die Entwicklung der schwedischen Politik der Bündnisfreiheit seit 1989 und auf die heutige Position des Landes eingegangen werden.

Wie die anderen bündnisfreien westeuropäischen Länder hat Schweden trotz der Beibehaltung dieses Status' seine Politik nach dem Ende des Kalten Krieges grundlegend geändert. Wiederkehrende Umformulierungen der schwedischen Definition ihrer Politik der Bündnisfreiheit spiegelten diesen Prozess wider. Ein großer Schritt wurde hierbei 2004 unternommen, als Schweden zum ersten Mal eine einseitige Erklärung formulierte, die später mehrmals wiederholt wurde. Die sogenannte, einseitige Solidaritätserklärung ‘ besagt, dass „Schweden nicht untätig bleiben wird, sollte ein anderes EU-Mitglied oder nordisches Land von einer Katastrophe oder einem Angriff getroffen werden. Gleichermaßen erwarten wir, dass diese Länder dasselbe tun, sollte eine ähnliche Krise Schweden treffen. Schweden sollte daher in der Lage sein, sowohl Unterstützung zu geben wie auch zu empfangen. ${ }^{2} 28$

26 Gemeinsame Erklärung des Prager Gipfeltreffens zur Östlichen Partnerschaft, Prag, 07.05.2009.

27 Swedish Presidency of the European Union: Chair's Conclusions, 08.12.2009.

28 Schwedische Regierung: Ett användbart försvar, Regeringens proposition 2008/09:140 [Eine funktionierende Verteidigung, Regierungsvorschlag 2008/09:140], 19.03.2009, S. 9. Das Parlament nahm den Vorschlag am 16. Juni 2009 an. Vertreter aller sieben Parteien erklärten später, dass sie die Erklärung unterstützen. Siehe auch Sten Tolgfors: A functional defence - with a substantially strengthened defence capability, Presseerklärung des Verteidigungsministers, 19.03.2009. 
Die einseitige Solidaritätserklärung sollte als Kodifizierung der momentan existierenden Lage interpretiert werden. In Übereinstimmung mit der Europäischen Sicherheitsstrategie (ESS) besagt die schwedische Ansicht, dass Bedrohungen nur gemeinsam begegnet werden kann. Wie gesagt, ist es unmöglich sich vorzustellen, dass jedweder Konflikt in der schwedischen Nachbarschaft nur ein Land allein betreffen kann. Die Interpretation des LissabonVertrages besagt, dass die Mitgliedstaaten jetzt solidarisch die Verantwortung für die Sicherheit Europas übernehmen. ${ }^{29}$ Die einseitige Solidaritätserklärung scheint die NATO-Mitgliedschaft zum logischen nächsten Schritt zu machen - vor allem, da Schweden seit den frühen 1990er Jahren gut mit der NATO zusammengearbeitet hat, einschließlich der Teilnahme an vielen NATO-geführten Friedensoperationen, aktuell in Afghanistan und dem Kosovo. Eine solche Entscheidung würde jedoch die Unterstützung der Bevölkerung benötigen. Die Unterstützung für eine Mitgliedschaft in der NATO ist in den letzten Jahren zwar gewachsen, doch die Zahl der Gegner einer solchen Mitgliedschaft ergibt, zusammen mit den Unentschiedenen, immer noch eine Mehrheit. ${ }^{30}$

Daraus folgt, dass Schweden in die sogenannte Solidaritätsklausel des Lissabon-Vertrages eingeschlossen ist, in der sich Mitgliedstaaten verpflichten andere Mitglieder, die von einem terroristischen Anschlag, einer Natur- oder sonstigen Katastrophe betroffen sind, zu unterstützen, (Art. 222 AEUV $^{31}$ ) aber von der Klausel der gegenseitigen Verteidigung mit dem Satz „Dies lässt den besonderen Charakter der Sicherheits- und Verteidigungspolitik bestimmter Mitgliedstaaten unberührt." befreit ist. (Art. 42 Abs. 7 EUV ${ }^{32}$ )

Die gegenwärtige Situation kann als paradox angesehen werden, wenn man die Gefühle des schwedischen Volkes zur Bündnisfreiheit außer Acht lässt, die nicht so sehr auf die Sicherheit abzielen, sondern mehr mit der eigenen Identität verknüpft werden. Gleichzeitig ist die NATO zeitweise mit einer nicht immer populären amerikanischen Politik in Verbindung gebracht worden. Solidarität mit europäischen Nachbarn ohne gleichzeitige Bündnisidentität kann insofern als eine gute Politik empfunden werden. Allerdings sollte daran erinnert werden, dass die Bevölkerung im Allgemeinen nur ziemlich geringes Interesse an und Wissen über diese Fragen hat. Die Solidaritätserklärung ist daher zurzeit zwar Gegenstand vieler Diskussionen, jedoch nur in akademischen und militärischen Kreisen und nur in Bezug darauf, ob schwedische Truppen in der Lage sind, anderen (das heißt den Baltischen Staaten) zu Hilfe kommen zu können, falls dies notwendig wird.

Schweden hat ein direktes Interesse daran, sowohl weltweit wie auch in Europa, an Sicherheitsfragen beteiligt zu werden. Beleg hierfür ist das langjährige Engagement bei den Friedensmissionen der Vereinten Nationen, die Teilnahme an allen ESVP-Missionen und Vorschläge für deren Entwicklung wie zum Beispiel die Petersberg-Aufgaben und das zivile Krisenmanagement. Dennoch kann der Status der Bündnisfreiheit den Eindruck eines geringeren Engagements vermitteln. Alle schwedischen Regierungen egal welcher Couleur stehen daher vor der Herausforderung zu zeigen, dass diese beiden Elemente kompatibel sind.

29 Ebenda.

302009 waren 35 Prozent für eine Mitgliedschaft Schwedens in der NATO (,sofort oder zu gegebener Zeit“), während 38 Prozent dagegen waren und 27 Prozent unentschieden. Siehe Myndigheten för samhällsskydd och beredskap (MSB): Opinioner 2009. Om allmänhetens syn på samhällsskydd, beredskap, säkerhetspolitik och försvar [Der Schwedische Zivilschutz (MSB): Meinungen 2009. Die Meinungen der Schweden über Gesellschaft, Sicherheit, Verteidigung und Zivilschutz], Stockholm 2009.

31 Vertrag über die Arbeitsweise der Europäischen Union, in: Amtsblatt der EU, Nr. C 83 vom 30. März 2010, S. 47-199.

32 Vertrag über die Europäische Union, in: Amtsblatt der EU, Nr. C 83 vom 30. März 2010, S. 13-45. 


\section{Vorschläge zur Europäischen Sicherheits- und Verteidigungspolitik}

Während der Ratspräsidentschaft legte Schweden vier Vorschläge zur Europäischen Sicherheits- und Verteidigungspolitik (ESVP) vor. Allen gemeinsam war der Versuch, die Effizienz und Koordination vorhandener Ressourcen zu verbessern statt neue einzubringen. Der erste Vorschlag betraf die Gefechtsverbände. Obwohl die ersten beiden Gefechtsverbände bereits seit Januar 2007 existieren und einsatzbereit sind, wurde keiner bisher eingesetzt. Ein besonderer Dorn im Auge Schwedens war die Situation im Frühjahr 2008, als klar wurde, dass der nordische Gefechtsverband nicht genutzt werden würde, obwohl gleichzeitig für die Tschad-Mission der Europäischen Union („EUFOR Tchad/RCA“) ${ }^{33}$ weitere Truppen benötigt wurden.

Schweden betrachtet die gegenwärtige Situation als große Verschwendung von Geld und Einsatzwillen und suchte daher eine Verständigung über eine Ausweitung der Einsatzfelder der Gefechtsverbände. Als Ergebnis dieser Bemühungen wurden die Regeln ergänzt und erlauben es jetzt, dass einer der Gefechtsverbände oder Teile davon außerhalb des etablierten Konzepts eingesetzt werden können, etwa als Reservetruppen oder als Teil der Truppenaufstellung (,force generation process') für eine bestimmte Operation. Derartige Entscheidungen werden von Fall zu Fall getroffen und erfordern zuallererst, dass die Länder, die zu diesem Gefechtsverband beitragen, dies so wollen. Eine andere Anforderung ist, dass diese Länder schon vor der Bereitstellung der Gefechtsverbände erklärt haben müssen, dass sie eine solche Flexibilität akzeptieren. Schließlich muss die Entscheidung über ihren Einsatz von allen EU-Mitgliedstaaten einstimmig getroffen werden. ${ }^{34}$ Dies dürfte schlussendlich den Ausschlag geben, da einige Staaten damit zufrieden sind, den Status quo beizubehalten.

Obwohl Schweden sich als einziges Land für dieses Thema stark machte, konnte das Land die Unterstützung anderer gewinnen, auch wenn der Militärausschuss der Europäischen Union und einige Länder vor zu weitgehenden Schritten warnen, weil sonst die Möglichkeit einer schnellen Reaktion verloren ginge. Auch die Frage der Finanzierung darf nicht außer Acht gelassen werden. Schweden ist gegen eine gemeinsame Finanzierung, weil diese, auch wenn sie einigen Ländern eine Beteiligung erleichtern würde, anderen eine weitere Begründung geben würde, eine Operation abzulehnen. ${ }^{35}$

Der zweite Vorschlag betraf die Europäische Verteidigungsagentur, deren Effizienz Schweden durch die Einführung eines offenen und transparenten europäischen Marktes für Rüstungsgüter stärken will, ohne Subventionen oder andere Einflussnahmen, von denen nationale Firmen profitieren könnten. Mehr als andere europäische Länder mit einer Rüstungsindustrie braucht Schweden einen fairen Markt, weil der eigene Markt klein ist. Der schwedische Verteidigungsminister Sten Tolgfors nahm für sich in Anspruch, dass die Antwort auf die schwedischen Argumente in dieser Angelegenheit positiv war. ${ }^{36}$

Die Integration ziviler und militärischer Fähigkeiten war ein dritter Schwerpunkt. Diese Initiative entsprang der Überzeugung, dass es notwendig sei, Synergien und Mehrfachnutzung in der Entwicklung der zivilen und militärischen Fähigkeiten der Europäischen Union

33 Hans-Georg Ehrhart: EUFOR Tchad/RCA: Zwischenbilanz und Empfehlungen für eine effektivere Konfliktlösung, in: integration 1/2009, S. 75-80; Hans-Georg Ehrhart: EU-Krisenmanagement in Afrika: die Operation EUFOR Tchad/RCA, in: integration 2/2008, S. 145-158.

34 Europe Diplomacy \& Defence. The Agence Europe Bulletin on ESDP and NATO, Nr. 266, 29.10.2009.

35 Verteidigungsminister Sten Tolgfors vor dem Parlamentsausschuss für europäische Angelegenheiten, 13. November 2009, S. 19-22.

36 Folk och Försvar [Gesellschaft und Verteidigung], Frukostseminarium med försvarsminister Sten Tolgfors [Frühstücksgespräch mit Verteidigungsminister Sten Tolgfors], 12.01.2010, Videoaufnahme abrufbar unter: http://bambuser.com/channel/folkochforsvar/broadcase/479693 (letzter Zugriff: 10.03.2010). 
zu identifizieren, um die Effizienz zu erhöhen. Zivile und militärische Fähigkeiten müssen gemeinsam entwickelt werden, um dem gleichen Ziel dienen zu können. Beispiele dafür sind das strategische Transportflugzeug „C-17“, das sowohl für zivile wie für militärische Zwecke, medizinische Hilfe und eine Vielzahl weiterer praktischer Bereiche, die im Laufe früherer Missionen erkannt wurden, eingesetzt werden kann. Eine neue Direktion ,Management und Planung', die im Ratssekretariat eingerichtet werden soll, wird hierbei eine wichtige Rolle spielen. In den Ratsbeschlüssen zur ESVP wird ferner die Integration der maritimen Überwachungssysteme (siehe unten) als konkretes Beispiel für zivil-militärische Synergien genannt. ${ }^{37}$ Als ein weiteres Beispiel für eine verbesserte zivil-militärische Zusammenarbeit kann die Kooperation der Vereinten Nationen, der Europäischen Union und der NATO gerade in Afghanistan angeführt werden. So wurden zum Treffen des Rates Allgemeine Angelegenheiten und Außenbeziehungen und zur gemeinsamen Tagung der Außenund der Entwicklungsminister am 16./17. November 2009 Kai Eide als Sonderbeauftragter der Vereinten Nationen für Afghanistan, Javier Solana als Generalsekretär des Rates/Hoher Vertreter für die Gemeinsame Außen- und Sicherheitspolitik und Anders Fogh Rasmussen als Generalsekretär der NATO zu den Beratungen eingeladen.

Die vierte Initiative, die im Herbst 2009 vorgestellt wurde, betraf die internationale $\mathrm{Zu}-$ sammenarbeit bei der Meeresüberwachung. Wie Verteidigungsminister Sten Tolgfors erklärte, entstand diese Idee aus Gesprächen mit seinem französischen Kollegen Hervé Morin, in denen sie darin übereinstimmten, dass die Europäische Union ein gemeinsames Informationssystem benötige, das auch das Geschehen in maritimen Bereichen abdeckt. Des Weiteren waren beide der Überzeugung, dass dies durch Interoperabilität, Zusammenarbeit und Informationsaustausch geschehen sollte. Vor Kurzem war das ostseeweite Seeraumüberwachungssystem „Sea Surveillance Cooperation Baltic Sea (SUCBAS)“ eingerichtet worden, das die nationalen Systeme der Ostseeanrainer verbindet. Das System ist ein Beispiel für sektor- und pfeilerübergreifende Zusammenarbeit, die aus den Herausforderungen wie organisiertes Verbrechen, Industrieunfälle oder Umweltkatastrophen, Terrorismus, Piraterie, illegale Einwanderung, illegales Fischen, Umweltverschmutzung und Auswirkungen des Klimawandels entstanden ist. Schweden erwartet, dass SUCBAS ein Modell und eine der Verbindungen sein wird, an die sich anderswo in Europa existierende Systeme anschließen, um einen Verbund zu schaffen, der die maritimen Bereiche abdeckt, die sowohl für zivile wie für militärische Zwecke nützlich sind. Das aktuelle Problem besteht also nicht im Fehlen solcher Systeme überhaupt, sondern in einem Mangel an Koordination und Informationsaustausch. ${ }^{38}$

\section{Die Europäische Union als Akteur der globalen Sicherheit}

Grundlage vieler Aktivitäten der Ratspräsidentschaft war der Anspruch, die Europäische Union als globalen Akteur weiterzubringen. Dies war ein selbstverständliches Anliegen angesichts der Tatsache, dass zwei der zentralen Probleme - die Finanzkrise und der Klima-

37 Europäische Sicherheits- und Verteidigungspolitik - Schlussfolgerungen des Rates, in: Rat der Europäischen Union: Mitteilung an die Presse. 2974. Tagung des Rates Allgemeine Angelegenheiten und Außenbeziehungen, Brüssel, den 17. November 2009, Dok. 15914/09 (Presse 329), S. 10; Verteidigungsminister Sten Tolgfors vor dem Parlamentsausschuss für europäische Angelegenheiten, 13. November 2009, S. 19.

38 Ebenda. Vollmitglieder sind zurzeit Dänemark, Deutschland, Estland, Finnland, Lettland, Litauen, Polen und Schweden. Russland hat keinerlei Interesse an einer Zusammenarbeit gezeigt. Siehe auch Europe Diplomacy \& Defence. The Agence Europe Bulletin on ESDP and NATO, Nr. 272, 24.11.2009; Rat der Europäischen Union: Bericht des Vorsitzes über die Integration der Meeresüberwachung, Dok. 15474/1/09; Anförande av försvarsminister Sten Tolgfors i Almedalen om Östersjösamarbetet [Rede von Verteidigungsminister Sten Tolgfors in Almedalen zur Ostseekooperation], Almedalen, Visby, 03.07.2009. 
wandel - globale Themen sind und globaler Lösungen bedürfen. Zusätzlich musste sich die Europäische Union einer langen Reihe von Sicherheitsfragen wie Afghanistan, Pakistan, Naher Osten, Iran und Nordkorea widmen, um dort zu Frieden und Stabilität beizutragen. Der Plan, die Rolle der Europäischen Union in Afghanistan und Pakistan zu stärken, schloss ein Maßnahmenpaket zur „Unterstützung der afghanischen und pakistanischen zivilen Kapazitäten, Verbesserung der politischen und demokratischen Reformen und verantwortlichem Regierungshandeln (, good governance'), Verbesserung der Sicherheitslage und sozioökonomische[n] Entwicklung“ ein. Während dieses Szenario entwickelt wurde, stellten Deutschland und Großbritannien unabhängig davon einen Vorschlag für eine Konferenz über die Zukunft Afghanistans vor, ohne diese Idee zuvor mit der Europäischen Union abzustimmen, was nach Aussage von Carl Bildt ,weiter diskutiert“ werden muss. ${ }^{39}$

Der Tradition, Kontakte auf globaler Ebene zu pflegen, folgend wurden während der Ratspräsidentschaft sieben Gipfeltreffen mit Ländern außerhalb der Europäischen Union organisiert. Die Priorität, die die Ratspräsidentschaft den Klimaproblemen zumaß, zeigte sich darin, dass alle Treffen sich mit Klima und Energie befassten. ${ }^{40}$ Weitere Themen wurden in anderen globalen Foren angesprochen, darunter Handelsfragen und Entwicklungshilfe. Die transatlantische Zusammenarbeit umfasste eine gemeinsame Initiative der Ratspräsidentschaft und der USA zur Weiterentwicklung in Bosnien, Verfassungsreformen eingeschlossen.

Weitere Aktivitäten im Zusammenhang mit der globalen Rolle der Europäischen Union wurden bereits angesprochen. Für die Nachbarschaft der Europäischen Union gilt dies außer für die bereits erwähnten Themen Erweiterung, Östliche Partnerschaft und europäisch-russischer Gipfel auch für Aktivitäten wie den Friedensprozess im Nahen Osten, die Union für das Mittelmeer und die Arktispolitik.

\section{Vorbereitungen für den Lissabon-Vertrag}

Zu Beginn der schwedischen Ratspräsidentschaft war nicht deutlich, ob der LissabonVertrag überhaupt in Kraft treten würde. Dennoch war es Aufgabe der Ratspräsidentschaft, Vorbereitungen für die reibungslose Umsetzung des Vertrages zu treffen, falls dies notwendig werden würde.

Die Arbeiten zum Europäischen Auswärtigen Dienst (EAD) wurden mit den Richtlinien der Ratspräsidentschaft präzisiert, die der Europäische Rat auf seinem Treffen am 29./30. Oktober 2009 beschloss. Es war Aufgabe der Ratspräsidentschaft, dem EAD einen Rahmen zu geben, den die Hohe Vertreterin der Union für Außen- und Sicherheitspolitik weiter ausfüllen sollte und, nach Beratungen mit dem Europäischen Parlament und der Zustimmung durch die Kommission, dem Europäischen Rat mit dem Ziel vorlegen wird, ihn bis spätestens Ende April 2010 zu beschließen. Der Bericht der Ratspräsidentschaft, dem Konsultationen mit den anderen 26 EU-Mitgliedstaaten vorausgegangen waren, skizziert eine Institution, die mit den diplomatischen Diensten der Mitgliedstaaten zusammenarbeiten soll und dessen Mitarbeiter aus dem Rat, der Kommission und den Mitgliedstaaten kommen sollen. Der EAD wird geografische und thematische Bereiche abdecken. Die Themenfelder Erweiterung wie auch Handel und Entwicklung werden in der Zuständigkeit der Kommission verbleiben. ${ }^{41}$

39 Swedish Presidency of the European Union: Strengthening EU action in Afghanistan and Pakistan, Fact Sheet, 27.10.2009; Carl Bildt vor dem Parlamentsausschuss für europäische Angelegenheiten, 11. September 2009, S. 13.

40 Die Länder waren Brasilien, China, Indien, Russland, Südafrika, die USA und die Ukraine.

41 Rat der Europäischen Union: Bericht des Vorsitzes an den Europäischen Rat über den Europäischen Auswärtigen Dienst, Dok. 14930/09; Rat der Europäischen Union: Tagung des Europäischen Rates am 29./30. Oktober 2009. Schlussfolgerungen des Vorsitzes, Dok. 15265/1/09, S. 2. 
Nach dem ,Ja'-Votum in Irland am 2. Oktober 2009 und der Ratifikation durch Polen (13. Oktober 2009) und Irland (23. Oktober 2009) war die Zustimmung des tschechischen Präsidenten, den Vertrag zu unterzeichnen, ein wichtiger Prüfstein für die Ratspräsidentschaft. Das Vorgehen erwies sich als ein Hindernislauf, da der tschechische Präsident immer wieder neue Hürden aufbaute, wie zum Beispiel eine ,opt-out' von der Menschenrechtscharta, die vom Europäischen Rat auf seinem Treffen am 29./30. Oktober 2009 zugestanden wurde. Die endgültige Unterzeichnung durch den tschechischen Präsidenten fand am 3. November 2009 statt, nachdem der tschechische Verfassungsgerichtshof erklärt hatte, dass der Lissabon-Vertrag im Einklang mit der tschechischen Verfassung stünde. Ein Telefonat zwischen Präsident Václav Klaus und Ministerpräsident Fredrik Reinfeldt, das Reinfeldt zu einem Zeitpunkt publik machte, als der tschechische Präsident seine Zweifel noch nicht öffentlich bekunden wollte, beschleunigte diesen Prozess außerordentlich. ${ }^{42}$

Nachdem die tschechische Unterschrift endlich unter Dach und Fach war, konnte Fredrik Reinfeldt mit den Konsultationen zu den Personalentscheidungen beginnen. Wie er vor dem schwedischen Parlamentsausschuss für europäische Angelegenheiten erläuterte, war dies eine schwierige Aufgabe. Der Präsident des Europäischen Rates würde aller Wahrscheinlichkeit nach eine Person sein, die an der Spitze der Regierung eines der 27 Mitgliedstaaten stand oder gestanden hatte. Wie im Fall des Hohen Vertreters der Union für Außen- und Sicherheitspolitik würden sich die Interessenten nicht öffentlich zu erkennen geben. Eine gescheiterte Kandidatur würde zu politischen Problemen in ihrem eigenen Land führen, da damit deutlich würde, dass sie bereit waren, ihr derzeitiges Amt aufzugeben. Reinfeldts Methode war, mit einem leeren Blatt Papier zu beginnen und jedes Land nach seinen Vorstellungen zu fragen. Er betrachtete es als seine Aufgabe sicherzustellen, dass jedes Land die Möglichkeit bekam, seine Kandidaten vorzuschlagen. Obwohl die qualifizierte Mehrheit für die Personalentscheidungen ausreichte, wollte er einen Konsens über die endgültigen Kandidaten erreichen. Auf die Frage nach seiner Arbeitsweise und dem Führungsstil erklärte er, dass er es bemerkenswert gefunden hätte, andere anzurufen und ihnen zu sagen, was sie denken sollten. Er sah diese Vorgehensweise als ein demokratisches Verfahren. Reinfeldt erläuterte, dass das wichtigste Kriterium bei der Kandidatensuche das politische Gleichgewicht zwischen einem Sozialisten und einem Nichtsozialisten für die beiden Positionen war. Erst danach wären andere Kriterien berücksichtigt worden: Kleinstaat gegen Großmacht, Ost gegen West und Nord gegen Süd und auch die Geschlechterfrage.

In der Frage, welcher Personentyp für die beiden Positionen geeignet wäre, verwies Reinfeldt auf die Existenz divergierender Ansichten in Europa einerseits sowie die Notwendigkeit, 27 souveräne Staaten miteinander in Einklang zu bringen, auf der anderen Seite war eine gemeinsame starke Stimme unerlässlich. Reinfeldts eigene Ansicht war, dass Europa sich nicht auf einen Namen festlegen sollte, der ein solches Extrem verkörperte, und er warnte vor allem davor, einen Politiker mit starken eigenen Vorstellungen zu fragen, der möglicherweise mit den Ansichten vieler Mitgliedstaaten im Konflikt stand. ${ }^{43}$

Mit dem Vorsatz, für die beiden Positionen einen Konsens zu erreichen, konsultierte Reinfeldt jedes Land zweimal und hatte mit einigen intensiven Kontakt. Dieser Prozess mündete in die Einigung vom 19. November 2009, den belgischen Premierminister Herman Van Rompuy als Präsidenten des Europäischen Rates und die britische Handelskommissarin Catherine Ashton als Hohe Vertreterin der Union für Außen- und Sicherheitspolitik/Vize-

42 Dagens Nyheter: Reinfeldt har retat upp Klaus [Reinfeldt hat Klaus verärgert], 11.10.2009.

43 Premierminister Fredrik Reinfeldt vor dem Parlamentsausschuss für europäische Angelegenheiten, 17. November 2009, S. 2-13. 
präsidentin der Kommission zu ernennen. Der Europäische Rat bestimmte zudem den bisherigen stellvertretenden Generalsekretär Pierre de Boissieu zum neuen Generalsekretär des Rates, die dritte wichtige Position, die zu besetzen war. Dahinter stand das Bedürfnis nach Stabilität in dieser Zeit großer Veränderungen. ${ }^{44}$

Ein weiterer Punkt von Bedeutung war die Notwendigkeit, Beschlüsse über wichtige Themen zu fassen, zu denen der Lissabon-Vertrag sich ausschwieg. Wie Reinfeldt gehofft hatte, fand der Europäische Rat bei seinem Treffen am 19. November 2009 auch Zeit, über seine Geschäftsordnung zu beraten. Es ging dabei um die Zusammenarbeit zwischen den Institutionen und Schlüsselakteuren, die neue und praktikable Formen benötigte. Von besonderer Bedeutung war es, die Rolle der rotierenden Ratspräsidentschaft neu zu bestimmen, die auch unter dem Lissabon-Vertrag noch eine wichtige Rolle spielt: Vorsitz bei den Ratstreffen und Verantwortung für die gesetzgeberische Arbeit des Rates. Die Ratspräsidentschaft erhielt auf diesem Treffen Unterstützung für ihre Arbeit und am 1. Dezember 2009 nahm der Europäische Rat seine neue Geschäftsordnung an. ${ }^{45}$

\section{Fazit - gute Noten für die schwedische Ratspräsidentschaft}

Bei seinem Treffen mit Europaparlamentariern am 16. Dezember 2009 erhielt der schwedische Ministerpräsident für die Ratspräsidentschaft seines Landes hohe Noten. José Manuel Barroso bezeichnete sie ebenfalls als sehr erfolgreich und als eine der besten der elf Ratspräsidentschaften, mit denen er bisher zusammengearbeitet habe. Bei den positiven Bewertungen stand an erster Stelle die Effizienz bei der Ausführung des versprochenen Programms, gefolgt von gut vorbereiteten Sitzungen und Transparenz der Verfahren. Außerdem wurde Fredrik Reinfeldt dafür gelobt, dass er die tschechische Unterschrift unter dem LissabonVertrag erreicht hatte.

Es gab aber auch einige negative Kommentare. Schweden habe nicht die Führungsrolle übernommen und habe auch nicht die erwartete Transparenz im Vorfeld der Ernennung von Van Rompuy und Ashton gezeigt. Stattdessen, so einige Kommentatoren, habe das Land den großen Staaten gestattet, diesen Prozess zu bestimmen und Personen auszuwählen, die von diesen Staaten dominiert werden würden. Andere negative Kommentare kamen von einigen Mitgliedern der Grünen und linken Parteien. Sie bezeichneten Schweden als wenig erfolgreich beim Thema Klimawandel. ${ }^{46}$

Die angesprochenen Prioritäten der schwedischen Ratspräsidentschaft folgen dem generellen Muster, dass sie überwiegend Erblasten von früheren Ratspräsidentschaften oder Teil der laufenden EU-Agenda waren. Dies betraf die Bereiche Finanzen und Arbeitsmarkt, das Stockholmer Programm im Bereich von Justiz und Inneres, die Klimafrage und die institutionellen Vorbereitungen. Die beiden anderen Themen entspringen eher den ureigenen schwedischen Interessen und Ambitionen: die EU-Strategie für den Ostseeraum, von der Schweden direkt profitiert, da es seine geografische Nachbarschaft betrifft, und die Arbeit zu den Themenkomplexen Erweiterung, Nachbarschaft und Sicherheit, der grundsätzlich viel Gewicht beigemessen wird.

44 Staatssekretär Gustaf Lind vor dem Parlamentsausschuss für europäische Angelegenheiten, 19. November 2009, S. 1-2; Beschluss des Europäischen Rates vom 1. Dezember 2009 zur Festlegung seiner Geschäftsordnung, in: Amtsblatt der EU, Nr. L 315 vom 2. Dezember 2009, S. 51-55.

45 Staatssekretär Gustaf Lind vor dem Parlamentsausschuss für europäische Angelegenheiten, 4. Dezember 2009, S. 2-9.

46 Rede des Premierministers Fredrik Reinfeldt vor dem Europäischen Parlament, in: Debates of the European Parliament Wednesday, 16 December 2009, S. 2-6. 
Die Tatsache, dass EU-Vorhaben ,geerbt" wurden, bedeutete jedoch nicht, dass sie automatisch nur als Routine behandelt wurden. Sowohl das Stockholmer Programm wie auch der Bericht zur nachhaltigen Entwicklungsstrategie spiegeln schwedische Anliegen wider. Auch wenn ihre Akzeptanz als Erfolg für Schweden gesehen werden kann, sollte daran erinnert werden, dass beide Dokumente eher Strategien als konkrete Beschlüsse darstellen. Es gibt also keine Garantie für ihre Umsetzung.

Die problemlose Akzeptanz der EU-Strategie für den Ostseeraum war positiv, doch der Wert dieser Strategie wird am Erfolg ihrer Umsetzung gemessen. Die Erweiterung hat Fortschritte gemacht und die Vorschläge zur ESVP könnten ebenfalls erfolgreich sein, auch wenn dies noch nicht abschließend beurteilt werden kann. Der vielleicht wichtigste Beitrag der Ratspräsidentschaft war die Art, mit der Schweden das Inkrafttreten des Lissabon-Vertrages ermöglichte: die Arbeit an den institutionellen Details, das vorsichtige Drängen gegenüber dem tschechischen Präsidenten und das gewagte Spiel mit der Veröffentlichung des Telefongesprächs. Bezüglich der abschließenden Verhandlungen über die beiden Spitzenpositionen ist es schwierig zu sagen, ob jene Kommentatoren Recht hatten, die sagten, dass die großen Länder sich durchgesetzt haben. Wie auch immer: Es ist kaum glaubhaft, dass nur Schweden aus dem Kreis der kleinen und mittelgroßen Länder sich vor einem Präsidenten mit einer starken eigenen Agenda hüten wollte.

Verbündete und Kooperationspartner wechselten je nach Tagesordnungspunkt. Frankreich war oft ein Partner in Fragen der ESVP, während Deutschland ein gleichgesinnter Verbündeter in Klimafragen und bei der EU-Strategie für den Ostseeraum war. Großbritannien teilte die schwedischen Ansichten zu Erweiterung und zu Handelsfragen. Die jeweilige Situation kann also auch die Wahl der Kooperationspartner bestimmen, so zum Beispiel in der Frage, wie mit dem Iran nach den Präsidentschaftswahlen umgegangen werden soll, wo Frankreich und Großbritannien wegen in Geiselhaft befindlicher Landsleute die engsten Mitstreiter waren. Die Zusammenarbeit mit EU-Institutionen scheint problemlos gewesen zu sein angesichts des Mangels an Beweisen für das Gegenteil und angesichts der im Allgemeinen positiven Kommentare von beiden Seiten.

\section{Ratspräsidentschaft und schwedische Innenpolitik}

Interne politische Angelegenheiten Schwedens hatten während der Ratspräsidentschaft wenig oder gar keine Bedeutung. Trotz der Vierparteienkoalition der Regierung (die moderate Partei Schwedens, Schwedens liberale Partei, Christdemokraten und Zentrumspartei) waren Trennlinien nicht wahrnehmbar, auch wenn Carl Bildt deutlich ein schärferes Profil hatte als die anderen Minister, wenn er sich mit Themen wie der Erweiterung befasste. Üblicherweise ist das schwedische Meinungsspektrum darüber, welche Politik Schweden innerhalb der Europäischen Union verfolgen soll, nicht breit gefächert. Im Parlamentsausschuss für europäische Angelegenheiten, in dem alle sieben Parteien vertreten sind, betraf Kritik während der Ratspräsidentschaft nur selten die Substanz der Politik. Eher wurde die Regierung dafür kritisiert, dass sie nicht genug für deren Ausführung täte.

Es ist schwer zu überprüfen, ob die Ratspräsidentschaft selbst die Ansichten über die Europäische Union in Schweden beeinflusst hat. Wie oben beschrieben gibt es seit langer Zeit eine fortdauernde Adaption von Ansichten und Politiken im Bereich der Sicherheit, da sich die Bedrohungen und Umstände für die europäische Sicherheit verändern. Die Unterstützung der Europäischen Union ist in der schwedischen Bevölkerung zwischen Frühjahr und Herbst 2009 leicht von 54 Prozent auf 57 Prozent gestiegen (EU-Mittelwert 53 Prozent). Es ist jedoch schwer zu sagen, ob dies an der Ratspräsidentschaft oder an etwas anderem gelegen hat. Auch die Akzeptanz des Euro ändert sich in eine positive Richtung: Im Dezember 
2009 waren 44 Prozent dafür und 42 Prozent dagegen. Dies ist allerdings ein Langzeittrend, der nicht mit der Ratspräsidentschaft in Verbindung gebracht werden kann. ${ }^{47}$

\section{Verschiedene Länder in der Position der Ratspräsidentschaft}

Wie soll die Rolle des Landes, das die Ratspräsidentschaft innehat, aussehen? Frankreich entschied sich während seiner Ratspräsidentschaft für eine starke Führungsrolle. Dieser französische Ansatz, gemeinsame Antworten auf die Finanzkrise zu finden, erhielt Lob wie auch die meisten der Aktivitäten im Georgien-Konflikt. Französischen Stimmen im Zusammenhang mit der eigenen Ratspräsidentschaft zufolge sollten nur große Länder die Ratspräsidentschaft übernehmen, da kleine Länder nicht in der Lage wären, diese anspruchsvolle Aufgabe zu schultern.

Das Lob für eine effiziente schwedische Ratspräsidentschaft scheint dieser Behauptung allerdings zu widersprechen. Anstelle politischer Führerschaft wurde Fredrik Reinfeldt eher die Rolle eines Koordinators attestiert und so war es in der Frage der Besetzung der beiden Spitzenpositionen offensichtlich auch seine Absicht gewesen. Es wäre sicherlich schwierig für ein kleines oder mittelgroßes Land, die gleiche Rolle wie ein großes Land zu spielen. Dies hängt nicht nur mit den kleinen Ländern und dem Gewicht, das sie in die Wagschale werfen können, zusammen, sondern auch damit, dass die großen Länder manchmal selbst aktiv werden statt sich mit dem Land, das den Vorsitz hat, abzusprechen. Während der schwedischen Ratspräsidentschaft war dies zum Beispiel bei der von Deutschland und Großbritannien organisierten Afghanistankonferenz der Fall und bei der von Frankreich organisierten Konferenz der Agrarminister am 10. Dezember 2009, fünf Tage vor dem Rat der EU-Agrarminister.

Eine andere gängige Meinung besagt, dass kleine Länder besser als große als ehrlicher Makler und Vermittler agieren können. Fredrik Reinfeldt beschrieb die schwedische Ratspräsidentschaft als eine ,zuhörende Präsidentschaft“", doch die kleineren Länder nur als ehrlichen Makler ohne eigene Interessen zu sehen, wird nicht von den schwedischen Aktivitäten und Ergebnissen gestützt. Die Ratspräsidentschaft scheint sich bemüht zu haben, der Europäischen Union den größtmöglichen eigenen Stempel aufzudrücken. Kleine Länder haben zugegebenermaßen nicht die gleichen globalen Interessen wie große und auch nicht die gleichen Möglichkeiten, diese zu verfolgen, aber Schweden (und sicherlich andere) hat versucht, seine Amtszeit dafür zu nutzen, die Union in die Richtung zu lenken, die es als die richtige erkannt hat. Ob ehrlich versucht wird, das zu verfolgen, was als das Beste für die Europäische Union oder nur für sich selbst erscheint, wird nicht von der Größe des Landes, sondern vom Charakter seiner Führung abhängen.

Der Verfasserin bleibt abschließend die Hoffnung, dass sich die EU-Mitgliedstaaten auf eine Weise zusammenfinden, die die Diskussion der Frage am Ende völlig unwichtig werden lässt. Noch fehlt der Union in diesem Punkt leider eine gemeinsame Vision und in einer Reihe von wichtigen Bereichen sind die 27 Mitgliedstaaten geteilter Ansicht. Der LissabonVertrag ist aus der Frustration über die Ineffizienz der Pfeilerstruktur entstanden und aus der Hoffnung auf ein besseres und einheitlicheres Funktionieren der Europäischen Union. Hoffentlich wird er jetzt dazu beitragen.

\section{Übersetzung aus dem Englischen von Gudrun Staedel-Schneider.}

47 Eurobarometer Nr. 72 zeigt, dass 57 Prozent die EU für, ,eine gute Sache“ halten, 25 Prozent finden sie „,weder gut noch schlecht“, 1 Prozent hat keine Meinung und 17 Prozent sagen, dass die EU ,eine schlechte Sache“ ist. Eurobarometer 72 Public Opinion in the European. First Results (Fieldwork: October-November 2009), Dezember 2009; Dagens Nyheter: Fler för än emot euron [Mehr für als gegen den Euro], 15.12.2009. 